\title{
Dynamics of a Rigid Test Body in Curved Space-Time*
}

\author{
H. P. KÜNZLE \\ Department of Mathematics, University of Alberta, Edmonton, Canada
}

Received February 23, 1972

\begin{abstract}
A covariant canonical formulation of the motion of a rigid test body in a curved space-time is obtained from a suitable Cartan form $\theta$ on the tangent bundle $T \mathscr{P}$ of the bundle of Lorentz frames $\mathscr{P}$ over the space-time manifold $V$. The form $\theta$ (essentially equivalent to a Lagrangean) is chosen in close analogy to the corresponding 1-form in the classical Newtonian model of a rigid body and is very simple in terms of the natural geometrical structure of $\mathscr{P}$. The presymplectic manifold $(T \mathscr{P}, d \theta)$ then serves as evolution manifold of the system. One obtains the equations of motion and also a uniquely defined Poisson bracket on the set of observables defined as real valued functions on the manifold of motions. The rigid body interacts with the space-time curvature only via its spin in the same way as a spinning particle. Quadrupole and higher multipole interactions with the space-time curvature are not considered in this model.
\end{abstract}

\section{Introduction}

The equations of motion of spinning particles, gyroscopes and rigid test bodies in curved space-time have been extensively discussed from different points of view (see mainly Suttorp and de Groot [25] and Dixon [5] for a historical review of the earlier literature). They have received added interest recently in view of the proposed gyroscope experiments $([21,8,17,18])$.

These equations have first been obtained by various generalizations of classical Newtonian equations which were, however, always somewhat arbitrary and lead to many controversies. Then Papapetrou [20] derived them from the conservation law for the stress-energy tensor of an extended body, an approach that was improved by Tulcyjew [27], Taub [26] and others until Dixon [5-7] and later more elegantly Madore [16] obtained a new and consistent set of equations, after the question of existence and uniqueness of a center of mass worldline was settled (Beiglböck [3]).

In this paper it is shown how Dixon's equations (specialized to a rigid test body freely falling in an exterior gravitational field, with quadrupole

* Supported in part by the National Research Council of Canada. 
and higher moments neglected) can be derived in a Lagrangean way. This may serve as a confirmation of the equations from a different point of view. The main purpose, however, is to show how in the modern framework of canonical dynamics (generalized Hamiltonian dynamics) the natural geometrical structures over space-time, like the principal bundle of Lorentz frames, with its given connection form and canonical form, can be used to find the general relativistic analogue of the classical rigid body system.

The modern theory of canonical dynamics is implicitly contained in several recent books on differential geometry and mechanics $([1,9,11])$, particularly in Souriau [23]. The precise definitions used here are given in [15]. For similar approaches and more details see [22] and [14].

Section 2 reviews the classical theory of the force-free rigid body in the modern formalism, following partly the treatment of Arnold [2]. The Cartan 1-form $\theta$ of the evolution manifold $\mathscr{E}=T S O(3) \times \mathbb{R}$ is expressed in terms of left invariant 1 -forms on $S O(3)$ and the rotational energy.

In Section 3 it is argued why the bundle of Lorentz frames $\mathscr{P}$ can serve as an (extended) configuration manifold for the relativistic rigid body. Since there is no convenient fibre coordinate system on $\mathscr{P}$, but on the other hand $\mathscr{P}$ is totally parallelizable (i.e., there is a natural isomorphism of $T \mathscr{P}$ and $\mathscr{P} \times \mathbb{R}^{10}$ ), we do not seek on $T \mathscr{P}$ an explicit Lagrangean, but only the corresponding Cartan 1-form $\theta$. This can be done in close analogy with the classical model of Section 2. Since the Lorentz group and hence any subgroup $S O(3)$ acts on the fibres of $\mathscr{P}$ we let the Killing vector fields of this action take the place of the left invariant vector fields on $S O(3)$, and similarly replace the left invariant 1 -forms by the corresponding components of the connection form on $\mathscr{P}$. The Cartan form $\theta$ on $\mathscr{P}$ can then be made to look formally almost the same as the one on $S O(3)$. Its exterior derivative $\omega:=d \theta$ defines a presymplectic structure on the 20 -dimensional manifold $T \mathscr{P}$, which is now chosen as the evolution manifold of the system.

The rest is straightforward computation. It is seen that $\operatorname{ker} \omega$ has dimension 8 such that the dimension of the motion manifold $\mathscr{M}:=T \mathscr{P} / \operatorname{ker} \omega$ becomes $20-8=12$ as is expected for a rigid body system. Moreover, the leaves of the foliation $\operatorname{ker} \omega$ on $T \mathscr{P}$ have well defined projections into $\mathscr{P}$ and space-time $\mathscr{V}$, which are easily described by the tensorial equations of the total and the translational motion, respectively. These equations agree with those of Dixon [7] for a dynamically rigid body provided quadrupole and higher multipole interactions of the body with the gravitational field are neglected. Note that the worldline of (the center of mass of) the body appears only at this stage. Contrary to many earlier other approaches our method does not use the 4-velocity in the definition of the configuration of the system. 
An attempt of a systematic study of the dynamical symmetries is made in Section 4, partly in order to note some other aspects of the formalism, partly to better interpret certain quantities physically.

\section{Canonical Form of the Classical Rigid Body System}

We consider in this section only the rotational motion of a rigid body in a framework similar to Arnold's [2]. This reformulation will give some guidance in the choice of the Cartan form in the relativistic case of the next section. The configuration manifold is the Lie group $\mathscr{G}=S O(3)$ and the motion as well as the symplectic structure of the force-free system are obtained from the Lagrangean

$$
L: T \mathscr{G} \rightarrow \mathbb{R}:(a, v) \mapsto L(a, v):=T_{\text {rot }}:=\frac{1}{2} I_{a}(v, v)
$$

where $I$ is a positive definite left invariant Riemannian metric on $\mathscr{G}$, i.e. satisfies $I_{a}=l_{a^{-1}}^{*} I_{e}$ where $I_{e}$ is the bilinear form on the Lie algebra $\mathfrak{g} \equiv T_{e} G$ and $l_{a}$ denotes the left translation by $a \in \mathscr{G}$. Classically $I$ is the tensor of inertia of the rigid body. We shall also consider $I_{a}$ as a linear isomorphism of $T_{a} \mathscr{G}$ onto $T_{a}^{*} \mathscr{G}$.

If, following the notation of Sternberg [24], we introduce the fibre derivative of the Lagrangean,

$$
\mathscr{L}: T \mathscr{G} \rightarrow T^{*} \mathscr{G}:(a, v) \mapsto\left(a, p=\frac{\partial L}{\partial v}=I_{a}(v)\right),
$$

the fundamental 1 -form $\theta_{0}:=-p_{\alpha} d a^{\alpha}$ on $T^{*} \mathscr{G}$ and the canonical symplectic form $\omega_{0}:=d \theta_{0}$, then the pullback of $\omega_{0}$ by $\mathscr{L}$ defines a symplectic structure on $T \mathscr{G}$.

However, since the tangent bundle of a Lie group is parallelizable in a natural way, it is more convenient not to work with the fibre coordinate systems $\left(a^{\alpha}, v^{\alpha}\right)$ and $\left(a^{\alpha}, p_{\alpha}\right)$ but rather with $\left(a^{\alpha}\right)$ and

or

$$
\Omega:=l_{a^{-1}}^{*} v \in \mathfrak{g}
$$

$$
S:=l_{a}^{*} p=l_{a}^{*} I_{a}(v)=I_{e}(\Omega) \in \mathfrak{g}^{*},
$$

respectively. Eqs. (1) and (2) exhibit the isomorphisms between $T \mathscr{G}$ and $\mathscr{G} \times \mathfrak{g}$ and between $T^{*} \mathscr{G}$ and $\mathscr{G} \times \mathfrak{g}^{*}$, respectively; $\Omega$ is called the angular velocity and $S$ the spin angular momentum or spin of the rigid body.

More explicitly, let $\left\{E_{A}\right\}$ be a basis of $\mathfrak{g},\left\{E^{A}\right\}$ the dual basis of $\mathfrak{g}^{*}$ and $E_{A}=E_{A}^{\alpha} \frac{\partial}{\partial a^{\alpha}}$ and $\tilde{E}^{A}=E_{\alpha}^{A} d a^{\alpha}$ the generated left invariant vector fields and 1 -forms on $\mathscr{G}$, respectively. Then, if we use the components 
$\Omega^{A}$ and $S_{A}$ of $\Omega$ and $S$ with respect to this basis, we find

and finally

$$
\begin{aligned}
& L=T_{\text {rot }}=\frac{1}{2} I_{A B} \Omega^{A} \Omega^{B}, \\
& \mathscr{L}:\left(a^{\alpha}, \Omega^{A}\right) \rightarrow\left(a^{\alpha}, S_{A}=I_{A B} \Omega^{B}\right), \\
& \theta_{0}=-p_{\alpha} E_{B}^{\alpha} \tilde{E}^{B}=-S_{A} E_{\alpha}^{A} E_{B}^{\alpha} \tilde{E}^{B}=-S_{A} \tilde{E}^{A}
\end{aligned}
$$

$$
\theta:=\mathscr{L}^{*} \theta_{0}=-\mathscr{L}^{*}\left(S_{B}\right) \tilde{E}^{B}=-I_{A B} \Omega^{A} \tilde{E}^{B},
$$

where $\left(I_{A B}\right)$ is always the constant symmetric positive definite $(3 \times 3)$ matrix of the components of the tensor of inertia with respect to a body fixed frame ${ }^{1}$.

Expressions for the symplectic forms on $\mathscr{G} \times \mathfrak{g}$ and $\mathscr{G} \times \mathfrak{g}^{*}$ follow by means of the Maurer-Cartan equations

$$
d \tilde{E}^{A}=-\frac{1}{2} C_{B C}^{A} \tilde{E}^{B} \wedge \tilde{E}^{C},
$$

where $C_{B C}^{A}$ are the structure constants of the Lie algebra g, i.e. $C_{B C}^{A}=\varepsilon^{A}{ }_{B C}$, the alternating symbol, in the case $\mathscr{G}=S O(3)$. One finds

$$
\omega=\frac{1}{2} S_{A B} \tilde{E}^{A} \wedge \tilde{E}^{B}+I_{A B} \tilde{E}^{A} \wedge d \Omega^{B},
$$

considered as a 2 -form on $\mathscr{G} \times \mathfrak{g}$ although the tensorial spin

$$
S_{A B}:=\varepsilon_{A B}{ }^{C} S_{C}=\varepsilon_{A B}{ }^{C} I_{C D} \Omega^{D}
$$

has been introduced for notational reasons.

The transition to the relativistic system will be somewhat easier if we first consider the corresponding contact structure on $T \mathscr{G} \times \mathbb{R}$. For our time-independent Lagrangean system the Cartan form is

$$
\bar{\theta}:=\operatorname{pr}_{1}^{*} \theta+\operatorname{pr}_{1}^{*}\left(T_{\text {rot }}\right) d t,
$$

where $\operatorname{pr}_{1}: T \mathscr{G} \times \mathbb{R} \rightarrow T \mathscr{G}$ is the canonical projection, and the presymplectic form is

$$
\bar{\omega}:=d \bar{\theta}=\frac{1}{2} S_{A B} \tilde{E}^{A} \wedge \tilde{E}^{B}+I_{A B} \tilde{E}^{A} \wedge d \Omega^{B}+S_{A} d \Omega^{A} \wedge d t .
$$

In this framework, the motions of the system are obtained as the set of leaves of $\operatorname{ker} \bar{\omega}$ on $T \mathscr{G} \times \mathbb{R}$. In terms of $\Omega^{A}$ one finds the classical Euler equations, namely,

$$
d \Omega^{A} / d t=-\tilde{I}^{A B} S_{B C} \Omega^{C}
$$

where $\left(\tilde{I}^{A B}\right)$ is the inverse of $\left(I_{A B}\right)$. In a principal frame for the tensor of inertia Eq. (8) become

$$
d \Omega^{1} / d t=I_{1}^{-1}\left(I_{2}-I_{3}\right) \Omega^{2} \Omega^{3}
$$

and the cyclically permuted equations for the other components.

\footnotetext{
${ }^{1}$ Capital latin indices will always run from 1 to 3 and their upper or lower position have no other meaning than to recall the summation convention.
} 


\section{A Relativistic Model}

For the freely moving rigid body in the non relativistic theory the total configuration manifold is $\mathbb{R}^{3} \times S O(3)$ and one chooses normally (see, for example [1,11,23 or 24]) as evolution manifold

$$
\mathscr{E}=\mathbb{R} \times T\left(\mathbb{R}^{3} \times S O(3)\right)
$$

with the Lagrangean

$$
L: \mathscr{E} \rightarrow \mathbb{R}:(t ; x, a ; v, \Omega) \mapsto \frac{1}{2} m v^{2}+\frac{1}{2} I_{A B} \Omega^{A} \Omega^{B} .
$$

The corresponding Cartan form $\theta$ on $\mathscr{E}$ is then

$$
\theta=-m v_{A} d x^{A}-I_{A B} \Omega^{A} \tilde{E}^{B}+\frac{1}{2}\left(m v_{A} v^{A}+I_{A B} \Omega^{A} \Omega^{B}\right) d t
$$

and it is easily verified that $d \theta$ has rank 12 thus defining a contact structure on the 13-dimensional manifold $\mathscr{E}$.

For relativistic systems it is well known that the formalism is more elegant if a so-called "extended" configuration manifold is used that includes the time among its dimensions. We generalize this idea somewhat as follows.

Let the space-time $\mathscr{V}$ be any time-oriented 4-dimensional Lorentz manifold. A first choice for an extended configuration manifold for the rigid body in $\mathscr{V}$ would seem to be any $S O(3)$-bundle over $\mathscr{V}$. However, no particular such bundle is distinguished, nor would it seem to exhibit any features of relativistic covariance.

On the other hand, the rotational configuration of a rigid body can also be described by a triad of orthonormal spacelike vectors $\left\{e_{A}\right\}$, thought of as directions of three body fixed axes at the center of mass of the body. Such a triad, at any $x \in \mathscr{V}$, however, determines precisely one future oriented Lorentz tetrad $\left\{e_{0}, e_{A}\right\}$ at $x$. In this sense there corresponds to every configuration "event" of the rigid body exactly one point $\left(x, e_{r}\right)^{2}$ of the bundle of Lorentz frames (for short, Lorentz bundle) $\mathscr{P}$ over $\mathscr{V}$. Since $\mathscr{P}$ also has a lot of geometrical structure, closely related to the space-time geometry it seems a good choice for the configuration manifold. Note that this approach avoids involving the 4-velocity of the body in the definition of its configuration which always seemed somewhat inconsistent.

As a principal fibre bundle $\mathscr{P}$ is totally parallelizable; in fact, there is a natural isomorphism between $T \mathscr{P}$ and $\mathscr{P} \times \mathbb{R}^{4} \times \mathfrak{g}$ where $g$ now stands for the Lie algebra of the Lorentz group. To make this isomorphism explicit let $\left\{E_{a b}, a<b\right\}$ be a basis of $\mathfrak{g}$. (If $\mathfrak{g}$ is represented by $(4 \times 4)$ -

2 Small Latin indices run from 0 to 3 and are raised and lowered with respect to the Minkowski metric $\eta:=\left(\eta_{a b}\right)=\operatorname{diag}(-1,1,1,1)$. 
matrices $A$ satisfying $A \cdot \eta+\eta \cdot A^{T}=0$ then $\left.\left(E_{a b}\right)_{k l}=\eta_{a k} \delta_{b l}-\delta_{a l} \eta_{b k}.\right)$ Then also

$$
E_{A}:=-\frac{1}{2} \varepsilon_{0 A}{ }^{r s} E_{r s}=-\frac{1}{2} \varepsilon_{A}{ }^{B C} E_{B C} \text { and } F_{A}:=E_{0 A}
$$

(where $\varepsilon_{a b c d}$ and $\varepsilon_{A B C}$ are the tensorial alternating symbols in Minkowski space and 3-dimensional Euclidean space, respectively) form a basis of $\mathfrak{g}$ and the $E_{A}$ generate a Lie subalgebra $\mathfrak{s o}(3)$ of $\mathfrak{g}$. The corresponding Killing vector fields $E_{A}$ and $F_{A}$ on $P$ of the group action together with the four standard horizontal vector fields $e_{r}$ (horizontal with respect to the connection induced by the Lorentz metric on $\mathscr{V}$ ) then form a basis of $T_{p} \mathscr{P}$ for every $p \in \mathscr{P}^{3}$. If we denote the (real valued) 1 -forms of the dual basis by $\left\{\theta^{r}, \tilde{E}^{A}, \tilde{F}^{A}\right\}$ the $\mathbb{R}^{4}$-valued canonical 1 -form $\boldsymbol{\theta}$ and the g-valued connection form $\boldsymbol{\omega}$ on $\mathscr{P}$ are explicitly $\boldsymbol{\theta}=\left(\theta^{0}, \theta^{1}, \theta^{2}, \theta^{3}\right)$ and $\omega=\tilde{E}^{A} E_{A}+\tilde{F}^{A} F_{A}$.

The arbitrary vector $X \in T_{p} \mathscr{P}$ can now be decomposed uniquely in the form $X=v^{r} \underline{r}_{r}+\Omega^{A} \underline{\sim}_{A}+U^{A}{\underset{\sim}{F}}_{A}$ and if we choose $\left(v^{r}, \Omega^{A}, U^{A}\right)$ as coordinates in $T_{p} \mathscr{P}$ we are in fact identifying $T \mathscr{P}$ with $\mathscr{P} \times\left(\mathbb{R}^{4} \times \mathfrak{g}\right)$ via the above mentioned isomorphism.

At this stage, noticing the similarity between the vector fields $E_{A}$ of this section with those of Section 2 (they generate an $S O(3)$ in both cases) we assume that expression (2.3) for the rotational energy in the body fixed system is still valid,

$$
T_{\text {rot }}:=\frac{1}{2} I_{A B} \Omega^{A} \Omega^{B},
$$

where we interpret the positive definite constant matrix $\left(I_{A B}\right)$ again as the given tensor of inertia of the body and $\Omega^{A}$ as the components of the angular velocity with respect to the body fixed frame $\left\{e_{A}\right\}$. Similarly we would expect the second term in (1) to carry over unchanged in notation.

As to the translational part of the Cartan form, the guessing is somewhat more difficult. As differentials we expect only the space-time coordinates to enter, that is only the 1-forms $\theta^{r}$. (Note that, in the fibre coordinate system $\left(x^{a}, e_{r}^{\alpha}\right), \theta^{r}=\theta_{\alpha}^{r} d x^{\alpha}$ with $\theta_{\alpha}^{r} e_{r}^{\beta}=\delta_{\alpha}^{\beta}, g_{\alpha \beta} e_{r}^{\alpha} e_{s}^{\beta}=\eta_{r s}$ and $e_{r}=e_{r}^{\alpha} \frac{\partial}{\partial x^{\alpha}}-\Gamma_{\beta \gamma}^{\alpha} e_{r}^{\beta} e_{c}^{\gamma} \frac{\partial}{\partial e_{c}^{\alpha}}$. $)$ We make the simplest possible choice (in our formalism) that reduces to the Newtonian case for flat space and small velocitites, namely we let the Cartan form be

$$
\theta=M \theta^{0}-I_{A B} \Omega^{A} \tilde{E}^{B}
$$

where $M:=m+T_{\text {rot }}, m$ being interpreted as the mass of the body as measured by a comoving observer when there is no rotation. This choice

\footnotetext{
${ }^{3}$ See, for example, Kobayashi/Nomizu [13], Chapter 3
} 
seems also quite reasonable in view of the fact that $\theta^{0}=d t$ if $t$ is the "proper" time of the frame $\left\{e_{A}\right\}$. We adopt now $\mathscr{E}=T \mathscr{P}$ as evolution manifold.

To find the induced presymplectic form $\omega:=d \theta$ the structure equations of the connection ${ }^{3}$ can now be used in place of the Maurer-Cartan equations. They become, if the structure constants of the Lorentz group are used,

$$
\begin{aligned}
& d \theta^{0}=\theta_{A} \wedge \tilde{F}^{A}, \\
& d \theta^{A}=\theta^{0} \wedge \tilde{F}^{A}-\varepsilon_{B C}^{A} \theta^{B} \wedge \tilde{E}^{C}, \\
& d \tilde{E}^{A}=\frac{1}{2} \varepsilon_{B C}^{A}\left(-\tilde{E}^{B} \wedge \tilde{E}^{C}+\tilde{F}^{B} \wedge \tilde{F}^{C}-\frac{1}{2} R^{B C}{ }_{r s} \theta^{r} \wedge \theta^{s}\right), \\
& d \tilde{F}^{A}=-\varepsilon_{B C}^{A} \tilde{E}^{B} \wedge \tilde{F}^{C}+\frac{1}{2} R_{0 r s}^{A} \theta^{r} \wedge \theta^{s} .
\end{aligned}
$$

From (3) to (7) and $d M=I_{A B} \Omega^{A} d \Omega^{B}=S_{A} d \Omega^{A}$ it then follows that

$$
\begin{aligned}
\omega= & \frac{1}{4} S^{A B} R_{A B r s} \theta^{r} \wedge \theta^{s}-S_{A} \theta^{0} \wedge d \Omega^{A}+M \theta_{A} \wedge \tilde{F}^{A} \\
& +\frac{1}{2} S_{A B}\left(\tilde{E}^{A} \wedge \tilde{E}^{B}-\tilde{F}^{A} \wedge \tilde{F}^{B}\right)+I_{A B} \tilde{E}^{A} \wedge d \Omega^{B}
\end{aligned}
$$

where $S_{A B}$ is again introduced by means of (2.6).

The next step is to investigate the kernel of $\omega$. Let

$$
Z=v^{r} \underline{v}_{r}+P^{A} \underbrace{}_{A}+Q^{A} \underline{F}_{A}+\xi^{r} \frac{\partial}{\partial v^{r}}+\Phi^{A} \frac{\partial}{\partial \Omega^{A}}+\Psi^{A} \frac{\partial}{\partial U^{A}}
$$

be any vector of $T_{q} \mathscr{E}$, then

$$
\begin{aligned}
Z\lrcorner \omega= & \left(S_{A} \Phi^{A}-\frac{1}{2} R_{0 r M N} v^{r} S^{M N}\right) \theta^{0}-\left(M Q_{A}+\frac{1}{2} R_{A r M N} v^{r} S^{M N}\right) \theta^{A} \\
& -\left(I_{A B} \Phi^{B}+S_{A B} P^{B}\right) \tilde{E}^{A}+\left(M v_{A}+S_{A B} Q^{B}\right) \tilde{F}^{A} \\
& +\left(I_{A B} P^{B}-v^{0} S_{A}\right) d \Omega^{A} .
\end{aligned}
$$

Therefore, $Z \in \operatorname{ker} \omega$ iff

$$
\begin{aligned}
P^{A} & =v^{O} \Omega^{A}, \\
\Phi^{A} & =-v^{0} \tilde{I}^{A B} S_{B C} \Omega^{C}, \\
M v^{A} & =-S^{A}{ }_{B} Q^{B}, \\
M Q^{A} & =-\frac{1}{2} R^{A}{ }_{r M N} v^{r} S^{M N}, \\
0 & =R_{0 r M N} v^{r} S^{M N} .
\end{aligned}
$$

Eq. (12) implies

$$
S_{A} v^{A}=0 .
$$

Then substitution of (13) into (12) and use of (15) yield

provided

$$
\begin{aligned}
& v^{A}=\frac{1}{2} M^{-2} \Delta^{-1} v^{0} S^{A B} R_{B O M N} \\
& \Delta:=1+\frac{1}{4} M^{-2} S^{A B} R_{A B C D} S^{C D} \neq 0 .
\end{aligned}
$$


Then (14) is trivially satisfied. The kernel of $\omega$ therefore consist of vectors $Z \in T_{q} \mathscr{E}$ whose components $v^{A}, P^{A}, Q^{A}$ and $\Phi^{A}$ are given by (16), (10, (13) and (11), respectively, in terms of $v^{0}$ and

$$
q=\left(x, e_{r} ; v^{r}, \Omega^{A}, U^{A}\right) \in \mathscr{P} \times\left(\mathbb{R}^{4} \times \mathfrak{g}\right)=\mathscr{E},
$$

while $v^{0}, \xi^{r}$ and $\Psi^{A}$ are arbitrary. Thus, $\operatorname{ker} \omega$ has dimension 8 . Since $\operatorname{ker} \omega$ is an integrable differentiable system it defines a foliation of $\mathscr{E}$. If this foliation is regular ${ }^{4}$ the quotient set $\mathscr{M}:=\mathscr{E} / \operatorname{ker} \omega$ is a 12-dimensional symplectic manifold, the "manifold of motions" of the rigid body system.

We now show that the projections of the leaves of $\operatorname{ker} \omega$ into $\mathscr{P}$ and $\mathscr{V}$ are well defined curves, the trajectories of the body in space-time and of its rotational motion. Let $\lambda$ be the parameter of an integral curve of a vector field $Z$ in $\operatorname{ker} \omega$. Then

$$
\left.\left.v^{\alpha}:=d x^{\alpha} / d \lambda=Z\right\lrcorner d x^{\alpha}=Z\right\lrcorner\left(e_{r}^{\alpha} \theta^{r}\right)=v^{r} e_{r}^{\alpha}
$$

is the tangent vector of the worldline of the body in space-time. If . $:=v^{\alpha} \nabla_{\alpha}$ denotes the covariant derivative along this worldline we find from

that

$$
\left.\left.d e_{r}^{\alpha} / d \lambda=Z\right\lrcorner d e_{r}^{\alpha}=Z\right\lrcorner\left(e_{s}^{\alpha} \tilde{E}_{r}^{s}-\Gamma_{\beta \gamma}^{\alpha} e_{r}^{\beta} e_{s}^{\gamma} \theta^{s}\right)
$$

and

and similarly

$$
\dot{e}_{0}^{\alpha}=e_{A}^{\alpha} Q^{A}
$$

Introduce now the tensor components of $\Omega$ and $S$ by

$$
\Omega^{\alpha}:=e_{A}^{\alpha} \Omega^{A}, \quad S^{\alpha}:=e_{A}^{\alpha} S^{A}
$$

and note that then

$$
S^{\alpha \beta}:=S^{A B} e_{A}^{\alpha} e_{B}^{\beta}=\varepsilon^{\alpha \beta}{ }_{\gamma \delta} e_{0}^{\gamma} S^{\delta} .
$$

Similarly let $\Omega_{\alpha \beta}:=\varepsilon_{\alpha \beta \gamma \delta} e_{0}^{\gamma} \Omega^{\delta}$. Now (18)-(21) are readily brought into completely tensorial form by substituting from (10), (11), (13) and (16). Thus the full set of equations of motion becomes

and

$$
\begin{gathered}
d x^{\alpha} / d \lambda=v^{\alpha}=v^{0}\left[e_{0}^{\alpha}+\frac{1}{2} M^{-2} \Delta^{-1} S^{\alpha \beta} R_{\beta \gamma \mu \nu} e_{0}^{\gamma} S^{\mu \nu}\right], \\
M \dot{e}_{0}^{\alpha}=-\frac{1}{2} R^{\alpha}{ }_{\beta \mu \nu} v^{\beta} S^{\mu \nu}, \\
\left(\delta_{\beta}^{\alpha}+e_{0}^{\alpha} e_{0 \beta}\right) \dot{e}_{A}^{\beta}=-v^{0} \Omega^{\alpha}{ }_{\beta} e_{A}^{\beta}
\end{gathered}
$$

$$
\left(\delta_{\beta}^{\alpha}+e_{0}^{\alpha} e_{0 \beta}\right) \dot{\Omega}^{\beta}=-v^{0} \tilde{I}^{\alpha \beta} S_{\beta \gamma} \Omega^{\gamma},
$$

${ }^{4}$ A global condition, not verifiable for a general space-time, see Palais [19] or Dieudonné [4]. 
where $\tilde{I}^{\alpha \beta}=\tilde{I}^{A B} e_{A}^{\alpha} e_{B}^{\beta}$, and $\Omega_{\alpha} e_{0}^{\alpha}=0=e_{0}^{\alpha} e_{A \alpha}$ has been used. The time development of $S^{\alpha}$ is determined by

$$
\left(\delta_{\beta}^{\alpha}+e_{0}^{\alpha} e_{0 \beta}\right) \dot{S}^{\beta}=0
$$

since again

$$
S_{x} e_{0}^{\alpha}=0 \text {. }
$$

It is easy to verify that the spin magnitude $s:=\sqrt{S^{\alpha} S_{\alpha}}$ and $T_{\text {rot }}$ and hence $M$ are constants of motion. Noting that $\delta_{\beta}^{\alpha}+e_{0}^{\alpha} e_{0 \beta}$ is the projection operator into the "rest space" spanned by $\left\{e_{A}\right\}$ makes it clear that (26), (27) and (28) are natural general relativistic analogues of the corresponding Newtonian equations. Together with (25) they are the equations found by Dixon [7]. Eq. (24) turns out to be a consequence of (25), (28) and (29). Clearly $v^{0}$ is determined by (24) if $\lambda$ is chosen to be the proper time along the worldline.

Finally, note that the condition (17) puts a limit on the spin magnitude for a given non zero space-time curvature. As remarked in [15], however, this is of no physical significance since the concept of test body is not valid for such large spins. Mathematically, it means that whenever space-time is not flat the evolution manifold is only some open submanifold of $T \mathscr{P}$.

\section{Observables and Symmetries}

While all real valued functions on the motion manifold $\mathscr{M}=\mathscr{E} / \operatorname{ker} \omega$ can be called observables or dynamical variables $([23,14])$ the only ones that can be readily and invariantly interpreted physically are those that arise from well defined group actions on $\mathscr{E}$ via Noether's theorem. One version of the latter states that when the vectorfield $A$ on $\mathscr{E}$ leaves the Cartan form $\theta$ invariant, that is, satisfies

then

$$
\mathscr{L}_{A} \theta=0
$$

$$
\left.f_{A}:=A\right\lrcorner \theta
$$

is an integral of motion, i.e. the pullback $f_{A}=\pi^{*} \tilde{f}_{A}$ of a function $\tilde{f}_{A}$ on $\mathscr{M}$. Since the group of all symmetries of $\theta$, being infinite dimensional, is too big to study and to interpret physically we confine ourselves to those vectorfields $A$ on $\mathscr{E}=T \mathscr{P}$ that satisfy (1) and have projections in $\mathscr{P}$ and in space-time $\mathscr{V}$. They clearly form a Lie algebra, generating what Hermann [10] calls the "obvious" symmetry group. This group turns out to be finite dimensional. In most cases it will coincide with the isometry group of space-time. 
Let $A$ be a general vectorfield on $\mathscr{E}$, again written in the form

$$
A=v^{r}{\underset{\sim}{r}}_{r}+P^{A}{\underset{\sim}{A}}_{A}+Q^{A}{\underset{\sim}{A}}_{A}+\xi^{r} \frac{\partial}{\partial v^{r}}+\Phi^{A} \frac{\partial}{\partial \Omega^{A}}+\Psi^{A} \frac{\partial}{\partial U^{A}} .
$$

Then $A$ has a projection in $\mathscr{P}$ provided $v^{r}, P^{A}$ and $Q^{A}$ do not depend on $v^{r}, \Omega^{A}$ or $U^{A}$. Substituting (3) into (1) gives

$$
\begin{aligned}
\left.\left.M v_{A}+S_{A B} Q^{B}+M\left(F_{A}\right\lrcorner d v^{0}\right)-S_{B}\left(F_{A}\right\lrcorner d P^{B}\right) & =0, \\
\left.\left.I_{A B} \Phi^{B}+S_{A B} P^{B}-M\left({\underset{\sim}{A}}_{A}\right\lrcorner d \nu^{0}\right)+S_{B}\left(E_{A}\right\lrcorner d P^{B}\right) & =0, \\
\left.\left.M Q_{A}+\frac{1}{2} R_{A r M N} v^{r} S^{M N}-M\left(e_{A}\right\lrcorner d v^{0}\right)+S_{B}\left(e_{A}\right\lrcorner d P^{B}\right) & =0, \\
\left.\left.S_{A} \Phi^{A}-\frac{1}{2} R_{0 r M N} v^{r} S^{M N}+M\left({\underset{\sim}{e}}_{0}\right\lrcorner d v^{0}\right)-S_{A}\left(\stackrel{e}{0}_{0}\right\lrcorner d P^{A}\right) & =0 .
\end{aligned}
$$

Thus, the part $\xi^{r} \partial / \partial v^{r}+\Psi^{A} \partial / \partial U^{A}$ remains completely arbitrary, but since this vector field is in $\operatorname{ker} \omega$ its projection into $\mathscr{M}$ vanishes and it does not contribute to the integral of motion $f_{A}$. The $\Phi^{A}$ 's are determined by (5) in terms of the components of $\tau_{\not \mathfrak{p} *} A$. Substituting them into (7) we find

$$
\begin{gathered}
\left.\left.-S_{A B} \Omega^{A} P^{B}-\frac{1}{2} R_{0 r M N} v^{r} S^{M N}+M\left(\Omega^{A}{\underset{E}{E}}_{A}\right\lrcorner d \nu^{0}+e_{0}\right\lrcorner d v^{0}\right) \\
\left.\left.-\Omega^{A}{\underset{\sim}{E}}_{A}\right\lrcorner d P^{B} S_{B}-{\underset{\sim}{0}}_{0}\right\lrcorner d P^{B} S_{B}=0 .
\end{gathered}
$$

Eqs. (4), (6) and (8) now constitute the conditions on $\tau_{\mathscr{P} *} A$ that must be satisfied for arbitrary $\Omega^{A}$. Setting the coefficients of all powers of $\Omega^{A}$ equal to zero leads to

and

$$
d v^{0}=Q_{A} \theta^{A}-v_{A} \tilde{F}^{A}
$$

where

$$
d P_{A}=\frac{1}{2} \varepsilon_{A M N} R^{M N}{ }_{r s} v^{r} \theta^{s}+K_{A B} \tilde{E}^{B}-\varepsilon_{A B C} Q^{B} \tilde{F}^{C},
$$

$$
K_{A B}=-\frac{1}{2} \varepsilon_{A B C} P^{C}+\frac{1}{2} \tilde{I}_{A}^{C} I_{B}^{D} \varepsilon_{C D E} P^{E}+\tilde{I}_{A}^{C} \varepsilon_{C B D} K^{D}
$$

where $K^{D}$ is still undetermined.

The condition that $A$ projects into $\mathscr{V}$ means that $v^{r}=v^{\alpha}(x) \theta_{\alpha}^{r}$, in terms of a fibre coordinate system. It follows that

and

$$
d v^{0}=\nabla_{r} v^{0} \theta^{r}-v_{A} \tilde{F}^{A}
$$

$$
d v^{A}=\nabla_{r} v^{A} \theta^{r}-v^{0} \tilde{F}^{A}+\varepsilon^{A}{ }_{B C} v^{B} \tilde{E}^{C}
$$

where $\nabla_{r} v^{s}$ are the tetrad components of the tensor $\nabla_{\alpha} v^{\beta}$ on $\mathscr{V}$. From (9) and (12) we see that $\nabla_{0} v^{0}=0$ and

$$
\nabla_{A} v^{0}=Q_{A} .
$$

Now take the exterior derivative of (9) and use (13) and (14) to find that

$$
\nabla_{(r} v_{s)}=0
$$

that is, $A$ must project onto a Killing vector field of the space-time metric. 
There remains to check the integrability conditions of (10), namely that the exterior derivative of the right hand side of (10) vanish. A longer calculation yields

$$
\begin{gathered}
d K_{A}=-\frac{1}{2} \varepsilon_{A B M} I_{N}^{B} R^{M N}{ }_{r s} v^{r} \theta^{s}-\lambda_{A B} \tilde{E}^{B}-\varepsilon_{A B[M} I_{N]}^{B} Q^{M} \tilde{F}^{N}, \\
K_{A B}=\nabla_{B} v_{A}
\end{gathered}
$$

and

where

$$
\begin{aligned}
0= & \varepsilon_{A M N}\left(\nabla_{[r} R_{s] k}{ }^{M N} v^{k}+V^{0} v^{M} R_{0}{ }^{N}{ }_{r s}\right)+\frac{1}{2} R_{A B r s} P^{B} \\
& +\frac{1}{4} \tilde{I}_{A}^{C} I_{B}^{D} \varepsilon^{B}{ }_{M N} \varepsilon_{C D E} P^{E} R^{M N}{ }_{r s}-\tilde{I}_{A B} K_{C} R^{B C}{ }_{r s}
\end{aligned}
$$

$$
\begin{aligned}
\lambda_{A B}=\frac{1}{2} I_{A}^{C} \varepsilon_{C B D} P^{D} & +\frac{1}{2} \varepsilon_{A C[B} I_{D]}^{C} P^{D}-\frac{1}{4} \tilde{I}_{A}^{C} I_{B}^{D} I_{D}^{E} \varepsilon_{C E F} P^{F} \\
& +\frac{1}{4} \varepsilon_{A K L} \varepsilon_{B M N} I^{K M} I^{L P} \varepsilon_{P Q R} \tilde{I}^{N Q} P^{R} \\
& +\varepsilon_{A B C} K^{C}-\tilde{I}_{A}^{C} I_{(B}^{D} \varepsilon_{Q) C D} K^{Q} .
\end{aligned}
$$

But from (11) and (17) we have

$$
K_{A}=\frac{1}{2} \varepsilon_{A B C} I_{D}^{B} \nabla^{C} v^{D} .
$$

The exterior derivative of (20) has the same coefficients of $\theta^{s}$ and $\tilde{F}^{N}$ as (16), but instead of $\lambda_{A B}$ the coefficient

$$
\tilde{\lambda}_{A B}=-\frac{1}{2} \nabla_{A} v^{C} I_{B C}-\frac{1}{2} \varepsilon_{A}{ }^{M N} \varepsilon_{B}{ }^{J}{ }^{K} I_{M K} \nabla_{J} v_{N} .
$$

Equating $\lambda_{A B}$ with $\tilde{\lambda}_{A B}$ is best done in a principal frame of $I_{A B}$. Whenever the tensor of inertia is not isotropic, i.e. $I_{A B} \neq I \delta_{A B}$ it follows that

$$
P^{A}=\frac{1}{2} \varepsilon^{A B C} \nabla_{B} v_{C} .
$$

In the isotropic case $P^{A}$ remains undetermined by this equation. However, (16), (17) and (10) imply in this case that $d\left(P^{A}+I K^{A}\right)=0$ whence

$$
P^{A}=\frac{1}{2} \varepsilon^{A B C} \nabla_{B} v_{C}+P_{0}^{A}
$$

where $P_{0}^{A}$ are three arbitrary constants. With all these results (18) now presents no further restriction.

The integrals of motion $f_{A}$ associated to these symmetries of the Cartan form are computed according to (2) and become

$$
f_{A}=M v^{0}-S_{A} P^{A}=M v^{0}-\frac{1}{2} S^{A B} \nabla_{A} v_{B}-S_{A} P_{0}^{A} .
$$

Thus the conclusion: The symmetries of the rigid body system that are lifts from the space-time $\mathscr{V}$ and the Lorentz bundle $\mathscr{P}$ are those which induce isometries of space-time in the non isotropic case. In the isotropic case there are three additional symmetries corresponding to the generators of the rotation group acting on the fibres of $\mathscr{P}$. The integrals of motion are

$$
f_{A}=-M v_{\alpha} e_{0}^{\alpha}-\frac{1}{2} S^{\alpha \beta} \nabla_{\alpha} v_{\beta}
$$

3 Commun math. Phys., Vol. 27 
where $v=v^{\alpha} \partial / \partial x^{\alpha}$ is a Killing vector field on $\mathscr{V}$. In the isotropic case the three additional integrals of motion are the components $S_{A}=S_{\alpha} e_{A}^{\alpha}$ of the spin vector.

For a Killing vector $v$ that is covariantly constant, that is, satisfies $\nabla_{\alpha} v_{\beta}=0$ the corresponding integral of motion is $f_{A}=-M v_{\alpha} e_{0}^{\alpha}$. If such an infinitesimal isometry can be considered as a generator of translations $f_{A}$ would be interpreted as the appropriate component of the 4-momentum. There is therefore some justification in calling $p^{\alpha}=M e_{0}^{\alpha}$ the 4-momentum vector of the rigid body, as has been done by Dixon $[5,7]$ and other authors from considerations involving the energy momentum tensor of an extended body.

\section{Conclusion}

We have confirmed Dixon's version of the equations of motion of a gyroscope in curved space-time from relatively simple, purely mechanical and geometrical considerations. New is the explicit construction of a symplectic structure on the motion manifold of this system.

In an earlier paper [15] the same aim was achieved for a spinning particle - a system with only four degrees of freedom. If one is only interested in the precession of the spin axis, rather than the whole rotational motion of the body - like in the case of a gyroscope rotating fast about an axis of symmetry - this previous model is good enough. Here we have shown that, in fact, as far as the motion of the spin axis is concerned the full rigid body model gives no new result.

While it is likely that the symplectic manifold of motions $(\mathscr{M}, \tilde{\omega})$ obtained here is the only reasonable relativistic analogue in curved spacetime of the Newtonian rigid body system, the choice of $T \mathscr{P}$ as evolution manifold is quite arbitrary. One can, for example, try to make $\mathscr{E}$ no bigger than necessary, that is restrict $\operatorname{dim} \mathscr{E}$ to $2 n+1$ if $n$ is the degree of freedom. An alternative approach that yields such a 13-dimensional evolution manifold with a contact structure by an application of the Dirac constraint algorithm is briefly outlined in an appendix, since, although less elegant, it has proved more useful in an application to the constrained motion of the rigid body.

\section{Appendix}

\section{An Alternative Approach}

The starting point is again $T \mathscr{P}$ equipped with the Cartan form $\theta$ as in (3.3). But we now require that a motion shall necessarily be an integral curve of a second order equation on the configuration manifold $\mathscr{P}$, that 
is of a vectorfield $Z$ on $T \mathscr{P}$ of the particular form

$$
Z=\chi\left(v^{r}{\underset{\sim}{e}}_{r}+\Omega^{A}{\underset{\sim}{A}}_{A}+U^{A}{\underset{\sim}{A}}_{A}\right)+\xi^{r} \frac{\partial}{\partial v^{r}}+\Phi^{A} \frac{\partial}{\partial \Omega^{A}}+\Psi^{A} \frac{\partial}{\partial U^{A}} .
$$

For such a vector field the equation $Z\lrcorner \omega=0$ cannot be solved on all of $T \mathscr{P}$. From (3.10) to (3.14) together with (1) we immediately find the conditions

and

$$
\begin{aligned}
& \Sigma^{0}:=v^{0}-1=0, \\
& \Sigma^{A}:=M v^{A}+S^{A}{ }_{B} U^{B}=0, \\
& T^{A}:=M U^{A}+\frac{1}{2} R_{r M N}^{A} v^{r} S^{M N}=0,
\end{aligned}
$$

$$
T^{0}:=R_{0 r M N} v^{r} S^{M N}=0 .
$$

The seven functions $\Sigma^{r}$ and $T^{A}$ turn out to be independent, while the last condition is a consequence of the other seven. Thus the vectorfield $Z$ is only defined on the 13-dimensional subbundle $\mathscr{N}$ of $T \mathscr{P}$, given by (2), (3) and (4). For consistency $Z$ must be tangent to $\mathscr{N}$, i.e. it must satisfy $\left.Z\lrcorner d \Sigma^{r}=0=Z\right\lrcorner d T^{A}$. These new seven equations are just enough to uniquely determine the components $\xi^{r}$ and $\Psi^{A}$ up to the arbitrary factor $\chi\left(\Phi^{A}\right.$ is still given by (3.11)).

Thus $\mathscr{N}$ can now be considered as the new evolution manifold of the system. According to a general theorem on such degenerate Lagrangean systems [14] the integral curves of $Z$ must coincide with the leaves of the foliation of $\operatorname{ker} \omega_{\mathscr{N}}$ on $\mathscr{N}$, where $\omega_{\mathscr{N}}:=l_{\mathscr{N}}^{*} \omega$ is the induced presymplectic form on the submanifold.

It is easy to verify that these integral curves are contained in the foliation defined by $\operatorname{ker} \omega$ on $T \mathscr{P}$ if they are considered as curves in $T \mathscr{P}$. Thus the two quotient manifolds $T \mathscr{P} / \operatorname{ker} \omega$ and $\mathscr{N} / \operatorname{ker} \omega_{\mathcal{N}}$ are diffeomorphic. Similarly the projections of the integral curves on $\mathscr{N}$ into $\mathscr{P}$ and $\mathscr{V}$ satisfy the equations obtained in Section 3, and also the study of the obvious symmetry group carries over almost literally.

\section{References}

1. Abraham, R., Marsden, J.E.: Foundations of mechanics. New York: Benjamin 1967.

2. Arnold, V.: Ann. Inst. Fourier, Grenoble 16, (1) 319--361 (1966).

3. Beiglböck, W. D.: Commun. math. Phys. 5, 106-130 (1967).

4. Dieudonné, J.: Eléments d'analyse, Vol. 3. Paris: Gauthier-Villars 1970.

5. Dixon, W. G.: Nuovo Cimento 34 (10), 317-339 (1964).

6. - Nuovo Cimento 38 (10), 1616-1643 (1965).

7. - Proc. Roy. Soc. Lond. A 314, 499-527 (1970).

8. Fairbank, W. M.: In: Allen, J. F., et al. (ed.): Proceedings of the Eleventh International Conference on Low Temperature Physics (St. Andrews Univ., 1969). 
9. Godbillon, C.: Géométrie différentielle et mécanique analytique. Paris: Hermann 1969.

10. Hermann, R.: Lie groups for physicists. New York: Benjamin 1966.

11. - Differential geometry and the calculus of variations. New York: Academic Press 1968.

12. - Lie algebras and quantum mechanics. New York: Benjamin 1966.

13. Kobayashi, S., Nomizu, K.: Foundations of differential geometry I. New York: Wiley 1963.

14. Künzle,H.P.: Ann. Inst. Henri Poincaré 11, 393-414 (1969).

15. - J. Math. Phys. 13, $739-744$ (1972).

16. Madore, J.: Ann. Inst. Henri Poincaré 11, 221-237 (1969).

17. - C. R. Acad. Sci. Paris 273 A, $782-784$ (1971).

18. O'Connell, R.F.: (1971) preprint

19. Palais, R. S.: Mem. Am. Math. Soc. 22 (Providence, 1957).

20. Papapetrou, A.: Proc. Roy. Soc. A 209, 248-258 (1951).

21. Schiff, L. I.: Proc. Nat. Acad. Sci. (U.S.) 46, 871-882 (1960).

22. Śniatycki, J., Tulczyjew, W. M.: Ann. Inst. Henri Poincaré 15, 177-187 (1971).

23. Souriau, J. M.: Structures des systèmes dynamiques. Paris: Dunod 1970.

24. Sternberg, S.: Lectures on differential geometry. Englewood Cliffs: Prentice Hall 1964.

25. Suttorp, L. G., Groot, S. R. de: Nuovo Cimento 65 A, 245-274 (1970).

26. Taub, A. H.: J. Math. Phys. 5, 112-119 (1964).

27. Tulczyjew, W.: Acta Phys. Polon. 18, 393-409 (1959).

H. P. Künzle

Dept. of Mathematics

University of Alberta

Edmonton, Alberta

T6G 2G1 